class is three-fourths of the half-wave length, and then in accordance with varying diameters above stated all larger diameters demand length to be increased in ratio, and more allowance to be made for the diverging than for the uniform bore; greater or less aniount of wind, and greater or less degree of pressure also enter into calculation, and practically are convertible in effect, the one doing duty for the other.

In the distinctive mode of action of this class we may find reasons for the varying relations of the pipes to each other, and for the contrasts shown in comparison with the other class acting under its own peculiar mnde. In these propulsive pipes in both the wide and narrow scales, the wind-current, after entering the foot of the pipe or boot, passes into the body of the pipe by a very contracted inlet formed by a hollow plug usually of metal called " the beak," or more commonly "the reed," to the confusion of inquirers; properly named, as we see it in old authors, it is "the shallot," from its resemblance in shape to the once favourite esculent the eschallot; ordinarily we speak of "the tongue " the elastic strip of metal covering it, as " the reed," for in the clarionet this part is really a reed. The main impulse of the current passes into the cone of the pipe through the mass of air in a central direction, and thus in a wide pipe, as compared with its course in a narrow pipe, the current has exchanged the friction upon the sides for the lesser friction of air upon air, still restricted, but less so in degree as the cone expands, as of a swift river escaping the confinement of banks, flooding the quiet expanding delta, agitating its waters with gradually-decreasing strength, and then becoming diffused in surrounding ocean.

Utmost exactness in length is quite as important for pitch and tone in these as in flue-pipes. Although the reed tongue has a determinate pitch of itself, yet a proper length of tube to reciprocate its action is indispensable, any inaccuracy only " upsets the tone," as the teclinical phrase says, and gives rise to curious freaks of behaviour. The slim tapering oboe is so sensitive that if we make it a quarter of an inch too long, or if we merely pat the top of its bell whilst sounding, the tone will immediately leap to its third above--not to a harmonic-a problem as puzzling as that of some echoes falling successively by thirds.

The action of the air-reed as causing suction by the velocity of passage of wind over the mouth was illustrated by me in a previous paper by reference to the abstracting power of a current of air, as shown in the spray-diffuser where globules of liquid are lifted and withdrawn by its agency. The action in these beating reeds is also susceptible of as simple an illustration. Take six or eight feet of india-rubber tubing of $\frac{1}{\mathrm{~s}}$-inch bore, for this length defines action more clearly - coil the length round your hand, and placing (ne end in the mouth blow through the tube sharply, at the same time allow the tip of your tongue freedom of play near the orifice, and you will find it drawn suddenly to the tube by the suction of the current passing down it, and released only on the exit of the current into the atmosphere. Lightly pressing the coil in your hand you may likewise feel the throb of the passing air-pulse. The trombone-player is conscious of his lip being forcibly drawn into the cup by a like cause. A stream of air suddenly cut off cannot pass down a tube without leaving a vacuum behind it. Organ and orchestral trumpets and oboes, and all of like propulsive action, are subject to this power, and only through it are able to generate tones. Suction is thus seen to be the final cause of vibration, the vacuum exists until the initial pulse of the vibration has made exit at the outer orifice, or in the second and succeeding courses until the pulses reach the colliding point or place of the prime node. Always thus in every musical pipe the current is essential to the suction, but with the striking difference that in the flue-pipe there is continuity of stream, and the continuity of flow is made available by conversion in reciprocating motion, but in the propulsive class the action is effective through discontinuity, by abrupt cessations and sequences of stream.

Here also in the beating-reed pipes we come upon distinct evidence of the interval of rest lengthening the period of vibration. The pitch of beating-reeds is regulated to consort with the pipe by means of a tuning-wire altering the vibrating length cf the tongue; thus regulated, the pitch may, however, within limited degree be altered by changing the force of wind, or by cutting off rim of pipe, or by adding thereto. Let it be observed that whether the tongue is pressed to the beak slowly or quickly, it will spring back in recoil in just the same time. By additional weight of wind, pitch may be raised, and in this case the tongue flies to more rapidly, but possibly any gain of speed in the advance may be counteracted by the recoil being impeded in the more compressed medium in which the tongue moves; the only remaining effect otherwise is that of an increased swiftness of the current of air more vigorously propelled in its course, and this in itself would account for the acceleration of pitch. On the other hand, leaving the force of wind constant, we may by temporary addition to the rim of upper orifice sensibly fiaten the pitch, for the current takes longer time to pass this extra boundary, hence the tongue is in consequence held longer upon the beak by the suction, its recoil delayed, or in other words recognising the physical result, its interval of rest is lengthened.

Many indications that come before me in my experiments lead directly to the inference that in all wind instruments this interval of rest is an important influence both on the pitch we regulate and in the quality we perceive ; and in the estimate I shall have to give of the interior process of working in the flue organ-pipe, I shall draw upon this inference that vibration is an activity tempered by rests.

One point has been unnoticed. It would be easy to find a diapason-pipe of the same pitch showing precise agreement in length with the trumpet above specified, and similarly for other various kinds. The recognition of numerous like correspond ences has led to the supposition that in relation to wave-length these two classes of pipes exhibit a parallelism. I hope to have made it clear that on the contrary, never parallel, the two classes proceed on two distinct systems of relation to wave-length, and are governed by a law, simply expressed as a law of divergent variation; they meet, it is true, but only at one point, where they cross in divergent lines, and they develop in opposite phases both in the ascending and descending extents of their range, the pitch of the one rising under an enlarging, and the other under a diminishing diameter.

Beyond the particular effects of friction already stated, the agency of the friction of air in the sound of wind instruments appears to me inadmissible. Reasons for this conclusion will occupy another paper in connection with details of my experiments bearing thereon under a simple device somewbat on the principle of the siren, and which may be named a "displacement siren."

Hermann SMITH

\section{Solar Halo}

ON Saturday last at Penruddock station, between Penrith and Keswick, about one o'clock, I observed a solar halo which at first was not perfect, but showed a reddish tint in the arc below the sun. Afterwards the circle became complete and continued so with small intervals until about half-past fuur, when I went indoors. At five o'clock the halo had disappeared in the haze. The day was thick, so liazy indeed that I could hardly distinguish the outline of Saddleback from Penruddock. The colour disappeared when the circle was complete, but occasionally I thought I could distinguish a reddish tinge on the inner side of the arc. I had no means of accurately measuring the radius, but with two pieces of stick which I picked up I estimated the tangent at $\frac{4}{3}$, which would give nearly $24^{\circ}$. This is more than your correspondent Mr. Gledhill found in his observations, but my measurement is confessedly rough. JAMES HEELIS

April 2I

\section{Saiety Matches}

Mr. TOMLINSON's remarks on safety-matches in NATURE, vol. xiii., p. 469 , reminded me that, not long ago I accidentally kindled one of those matches by rubbing it on the edge of a Wedgwood-ware morlar. This material appears even better adapted than those mentioned by Mr. Tomlinson for igniting such matches, and $I$ found that a common earthenware dish (glazed inside) answered the same purpose admirably. I tried to ascertain the degree of certainty with which a safety-match could be kindled by friction against these two materials, and was surprised to find that they are little inferior in this respect to amorphous phosphorus itself. After a little practice in the manner of striking, it is easy to kindle nearly every match. Thus $I$ have lighted forty matches out of fourty-four (most of them at the first or second stroke), using the glazed portion of the basin referred to. I should add that the surface becomes improved by use, which can hardly he said of the composition on the sides of the safety-match boxes.

Manchester, April I8

FranCIS JONES

\section{"The Ash Seed Screw"}

THE delicate twist in the samara of the ash is clearly not that best calculated to retard descent. The more decided the twist, 